\title{
Novel link between E2F and p53: proapoptotic cofactors of p53 are transcriptionally upregulated by E2F
}

\author{
T Hershko ${ }^{1}$, M Chaussepied $^{1}$, M Oren ${ }^{1}$ and D Ginsberg ${ }^{*, 1}$ \\ ${ }^{1}$ Department of Molecular Cell Biology, The Weizmann Institute of Science, \\ Rehovot 76100, Israel \\ * Corresponding author: D Ginsberg, Department of Molecular Cell Biology, The \\ Weizmann Institute of Science, Hertzel St., Rehovot 76100, Israel. \\ Tel: 9728 9342239; Fax: 9728 9344125; \\ E-mail: doron.ginsberg@weizmann.ac.il
}

Received 29.11.04; revised 17.12.04; accepted 17.12.04; published online 11.02.05 Edited by G Melino

\begin{abstract}
The E2F1 transcription factor is a critical downstream target of the tumor suppressor RB. When activated, E2F1 induces cell proliferation. In addition, E2F1 can induce apoptosis via both p53-dependent and p53-independent pathways. A number of E2F-regulated genes, including ARF, ATM and Chk2, contribute to E2F-induced p53 stabilization. However, it is not known how E2F directs p53 activity towards apoptosis rather than growth arrest. We show that E2F1 upregulates the expression of four proapoptotic cofactors of p53 - ASPP1, ASPP2, JMY and TP53INP1 - through a direct transcriptional mechanism. Adenovirus E1A protein also induces upregulation of these genes, implicating endogenous E2F in this effect. TP53INP1 was shown to mediate phosphorylation of p53 on serine 46. We demonstrate that activation of E2F1 leads to phosphorylation of p53 on serine 46 and this modification is important for E2F1-p53 cooperation in apoptosis. Overall, these data provide novel functional links between RB/E2F pathway and p53-induced apoptosis.

Cell Death and Differentiation (2005) 12, 377-383.

doi:10.1038/sj.cdd.4401575

Published online 11 February 2005
\end{abstract}

Keywords: E2F; p53; apoptosis; ASPP family; TP53INP1; JMY

Abbreviations: ChIP, chromatin immunoprecipitation; $\mathrm{CHX}$ cycloheximide; ER, estrogen receptor; FCS, fetal calf serum; OHT, 4-hydroxytamoxifen

\section{Introduction}

The E2F family of transcription factors plays a critical role in the control of cell proliferation by regulating the timely expression of many genes required for cell cycle progression (reviewed in DeGregori ${ }^{1}$ ). At least one member of this family, E2F1, can also mediate apoptosis (reviewed in Ginsberg ${ }^{2}$ ). Ectopic expression of E2F1 leads to apoptosis in tissue culture cells $\mathrm{s}^{3-5}$ and transgenic mice ${ }^{6-8}$ Moreover, a physio- logical role for E2F1-mediated apoptosis is suggested by the observation that mice deficient in E2F1 have an excess of mature T cells due to a defect in thymocyte apoptosis. ${ }^{9}$ E2F1induced apoptosis occurs via both p53-dependent and -independent pathways. ${ }^{2}$ E2F1 signaling to p53 is partially mediated by $\mathrm{p} 14^{\mathrm{ARF}}$, which is transcriptionally activated by E2F and encodes a protein that stabilizes and activates p53 by negating the effects of the E3 ubiquitin ligase Mdm2 on p53 (reviewed in Sherr and Weber ${ }^{10}$ ). However, E2F1 has been shown to induce p53-dependent apoptosis in ARF-deficient mice and cells, ${ }^{11-14}$ indicating that there are additional, ARFindependent, functional links between E2F1 and p53. E2F1 can induce phosphorylation of p53 on residues that are phosphorylated in response to DNA damage ${ }^{11,15,16}$ and, in agreement with these observations, E2F1 transcriptionally regulates the expression of the DNA damage-responsive kinases ATM and Chk2 that phosphorylate p53. ${ }^{15,17}$ Consistent with the ability of both ARF and the DNA damage-induced kinases to cause p53 stabilization, ectopic expression of E2F1 indeed induces p53 accumulation. ${ }^{5,18}$

p53 regulates the transcription of many genes that induce cell cycle arrest or apoptosis in response to DNA damage or oncogenic stress. ${ }^{19}$ Till recently, it was not clear how p53 'decides' whether to cause growth arrest or apoptosis. Some insights into this decision-making process come from the isolation and characterization of p53 regulators such as ASPP1, ASPP2, ${ }^{20} \mathrm{JMY}^{21}$ and TP53INP1(also named p53DINP1 and SIP). ${ }^{22}$ These proteins direct p53 to specifically favor the activation of proapoptotic target genes over growth arrest-related target genes. They are, therefore, considered to be proapoptotic cofactors of $\mathrm{p} 53$. The cofactors affect p53 activity via distinct mechanisms: Members of the apoptosis stimulating proteins of p53 (ASPP) family, ASPP1 and ASPP 2 , interact with $\mathrm{p} 53$ and enhance its interaction with the promoter of its apoptotic target, BAX, thereby enhancing p53-induced apoptosis. ${ }^{20}$ Another member of the ASPP family, iASPP, antagonizes ASPP1 and ASPP2: it was shown to inhibit p53-induced apoptosis and its depletion induces p53-dependent apoptosis. ${ }^{23,24}$

JMY, another proapoptotic cofactor of p53, interacts with p300, which functions as a coactivator for p53, thereby enhancing the ability of p53 to induce the expression of genes such as BAX, without significantly influencing the induction of p21. ${ }^{21}$ The net effect of this is that JMY promotes the induction of apoptosis, but not cell-cycle arrest by p53.21

Tumor suppressor p53-induced nuclear protein 1 (TP53INP1) was cloned independently from human and mouse cells, and was named p53DINP1 ${ }^{22}$ and $\mathrm{SIP}^{25}$, respectively. TP53INP1 was shown to be a p53-regulated gene. ${ }^{22,26}$ The TP53INP1 protein interacts with $\mathrm{p} 53^{27}$ and with a kinase that phosphorylates $p 53$ on serine $46,{ }^{22,27}$ thereby inducing phosphorylation of $p 53$ at this site. ${ }^{22}$ This modified p53 plays a pivotal role in apoptotic signaling, mainly through 
regulating the transcriptional activation of p53AIP1, an important mediator of p53-dependent apoptosis. ${ }^{22}$ Indeed, ectopic expression of TP53INP1 triggers apoptosis ${ }^{25}$ and enhances p53-induced apoptosis. ${ }^{22}$

We show here that excessive E2F activity, achieved either by ectopic expression of E2F1 or by disruption of the RB/E2F complexes, results in increased expression of ASPP1, ASPP2, JMY and TP53INP1. Transcription of all four genes is directly regulated by $\mathrm{E} 2 \mathrm{~F}$, which binds their promoters in vivo. Furthermore, E2F1 induces phosphorylation of p53 on serine 46 and this modification is important for E2F1-p53 cooperation in apoptosis. These findings delineate a novel mechanism for the cooperation of E2F1 and p53 in apoptosis.

\section{Results}

To determine whether E2F regulates the expression of proapoptotic cofactors of p53, we examined the effect of E2F1 on expression of four such cofactors - ASPP1, ASPP2, JMY and TP53INP1. Introduction of E2F1 into U2OS human osteosarcoma cells by retrovirus-mediated gene transfer resulted in a significant increase in the endogenous mRNA levels of all four genes (Figure 1a). Human TP53INP1 has two alternatively spliced isoforms ${ }^{22}$ and both were induced by E2F1 (Figure 1a). Next, we infected WI38 human embryonic lung fibroblasts with a retrovirus expressing a conditionally active E2F1, ER-E2F1. ${ }^{28}$ Activation of E2F1 by addition of
OHT to these cells led to an increase in mRNA levels of ASPP1, ASPP2, JMY and TP53INP1 (Figure 1b). The positive effect of E2F1 on the expression of p53 proapoptotic cofactors was also confirmed for the corresponding proteins: activation of ER-E2F1 in U2OS cells augmented the endogenous JMY protein levels (Figure 1c). Furthermore, induction of ER-E2F1 in WI38 cells led to an increase in ASPP1 protein (Figure 1d). Of note, a DNA binding-deficient mutant of E2F1 (E2F1 E132) failed to induce accumulation of JMY and ASPP1 (Figure 1c and $\mathrm{d}$ ), despite being expressed at comparable levels to wt E2F1 (data not shown), implying that the effect of E2F1 is exerted through a transcription-dependent mechanism.

To study the regulation of expression of p53 proapoptotic cofactors by endogenous E2F, we infected WI38 cells with a retrovirus expressing the adenoviral oncoprotein $E 1 A$, which disrupts RB/E2F complexes, thereby leading to deregulation of endogenous E2F activity. Expression of E1A led to a significant increase in ASPP1, ASPP2, JMY and TP53INP1 mRNA (Figure 1e), strongly suggesting that deregulated endogenous E2F transcriptionally activates all these genes.

The TP53INP1 gene is positively regulated by $p 53 .^{22}$ E2F1 can upregulate p53 levels ${ }^{5,18}$ and, therefore, the possible involvement of $\mathrm{p} 53$ in E2F1-induced upregulation of TP53INP1 expression was tested using p53-deficient cells. Expression of either E2F1 or E1A in p53-null human $\mathrm{H} 1299$ lung adenocarcinoma cells resulted in an increase in TP53INP1 mRNA (Figure 2a), indicating that this increase can occur in a p53-independent manner.
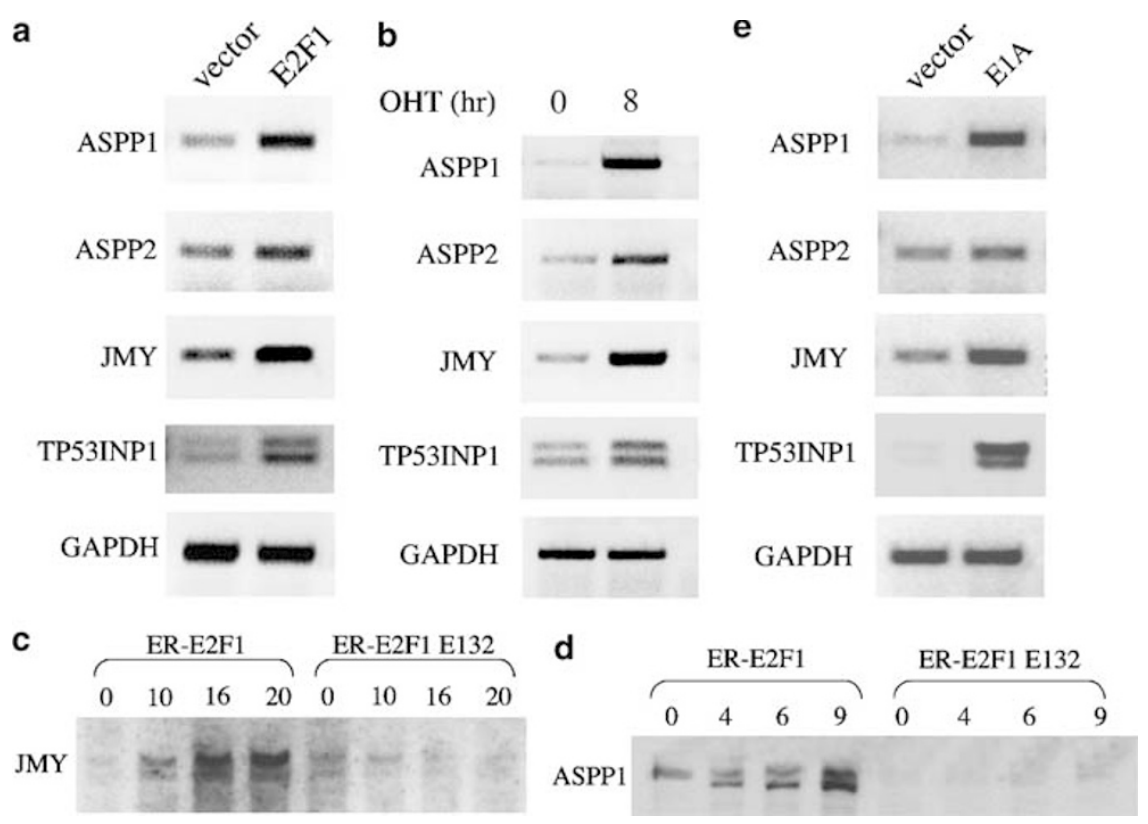

Figure 1 E2F1 upregulates ASPP1, ASPP2, JMY and TP53INP1 levels. (a) U2OS cells were infected with a control retrovirus (vector) or a retrovirus expressing E2F1 (E2F1). At $24 \mathrm{~h}$ post infection, puromycin was added to the culture for $24 \mathrm{~h}$. Total RNA was extracted from the cells and RT-PCR analysis was performed using primers specific for ASPP1, ASPP2, JMY, TP53INP1 and GAPDH. (b) WI38 cells containing ER-E2F1 were incubated with or without OHT ( $300 \mathrm{nM}$ ) for $8 \mathrm{~h}$. Total RNA was extracted from the cells and RT-PCR analysis was performed using primers specific for ASPP1, ASPP2, JMY, TP53INP1 and GAPDH. (c) U2OS cells were infected with a retrovirus expressing either ER-wild type E2F1 (ER-E2F1) or a retrovirus expressing ER-E2F1-E132. Infected cells were incubated with $\mathrm{OHT}$ ( $300 \mathrm{nM}$ ) for the times indicated at the top of each lane. Proteins were extracted from the cells and equal amounts of protein (determined by Bradford assay) were used for Western blot analysis with an anti-JMY antibody (sc-10027). (d) WI38 cells were infected with a retrovirus expressing either ER-wild type E2F1 (ER-E2F1) or a retrovirus expressing ER-E2F1-E132. Infected cells were incubated with $\mathrm{OHT}(300 \mathrm{nM})$ for the times indicated at the top of each lane. Proteins were extracted from the cells and equal amounts of protein (determined by Bradford assay) were used for Western blot analysis with an anti-ASPP1 antibody (generous gift of Xin Lu). (e) WI38 cells were infected with a control retrovirus (vector) or a retrovirus expressing E1A (E1A). Total RNA was extracted from the cells and RT-PCR analysis was performed using specific primers for ASPP1, ASPP2, JMY, TP53INP1 and GAPDH 
a

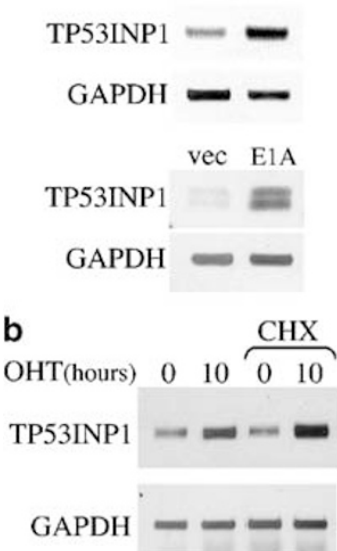

C
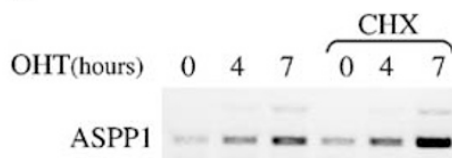

ASPP2 - - - -

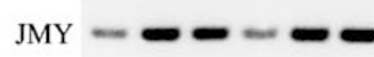

GAPDH $---m-$

Figure 2 ASPP1, ASPP2, JMY and TP53INP1 are direct E2F targets. (a) H1299 cells were infected with a control retrovirus (vector) or a retrovirus expressing either E2F1 (upper panels) or E1A (lower panels). Total RNA was extracted from the cells and RT-PCR analysis was performed using primers specific for TP53INP1 and GAPDH. (b, c) U20S cells containing the ER-E2F1 fusion protein were incubated with $\mathrm{OHT}$ for the times indicated at the top of each lane. Where indicated, $\mathrm{CHX}$ was added for $4 \mathrm{~h}$ prior to harvesting. Total RNA was extracted from the cells and RT-PCR analysis was performed using specific primers for ASPP1, ASPP2, JMY and GAPDH genes (b) or the TP53INP1 and GAPDH (c)

The E2F1-induced increase in mRNA levels of the four p53 proapoptotic cofactors was detected also in the presence of the protein synthesis inhibitor cycloheximide (CHX) (Figure $2 b$ and $\mathrm{c}$ ). Hence, de novo protein synthesis is not required for E2F1-mediated regulation of these four genes, suggesting that they are direct E2F targets. Interestingly, expression of these genes was induced with different kinetics: while induction of TP53INP1 was detectable only 8-10 h after E2F1 activation (Figure 2b), expression of ASPP1, ASPP2 and JMY was affected by E2F1 already $4 \mathrm{~h}$ after addition of OHT (Figure 2c).

To date, the promoters of these genes have not been functionally characterized. However, examination of the human genomic sequences spanning 1000 base pairs upstream of the putative transcription start site of each of the four target genes (i.e. the $5^{\prime}$ end of the longest reported cDNA), using the Matlnspector program (release 6.2.2), revealed the presence of putative E2F-binding sites in the promoter regions of ASPP1, ASPP2 and TP53INP1 (Figure 3a): in ASPP1, one site at position -762/-769 (GCGGGAGA); in ASPP2, four sites at positions -130/ -139 (GTTCCCGC), -426/-435 (GCGCCCAA), -656/ -665 (ACGGCAAA) and -993/-1002 (GTTCCCGC); and in TP53INP1, one site at position -342/351 (TTGCCCGC) relative to the transcription start site. No consensus E2Fbinding site could be detected in the human JMY promoter; however, various promoters have been shown to recruit E2F via a mechanism independent of the defined consensus site. $^{29}$

To further demonstrate the involvement of endogenous E2Fs in regulating the expression of these E2F-responsive genes, we performed a chromatin immunoprecipitation analysis (ChIP) using chromatin from proliferating WI38 human embryonic lung fibroblasts and antibodies directed

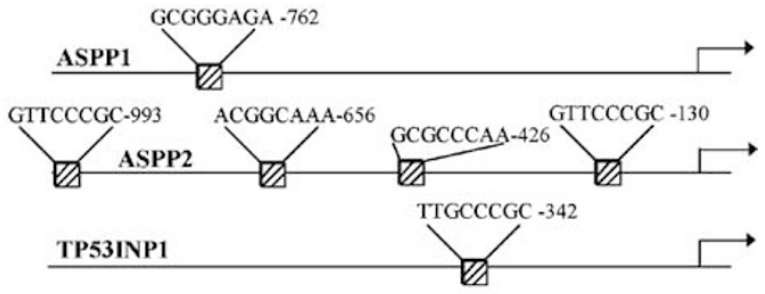

b

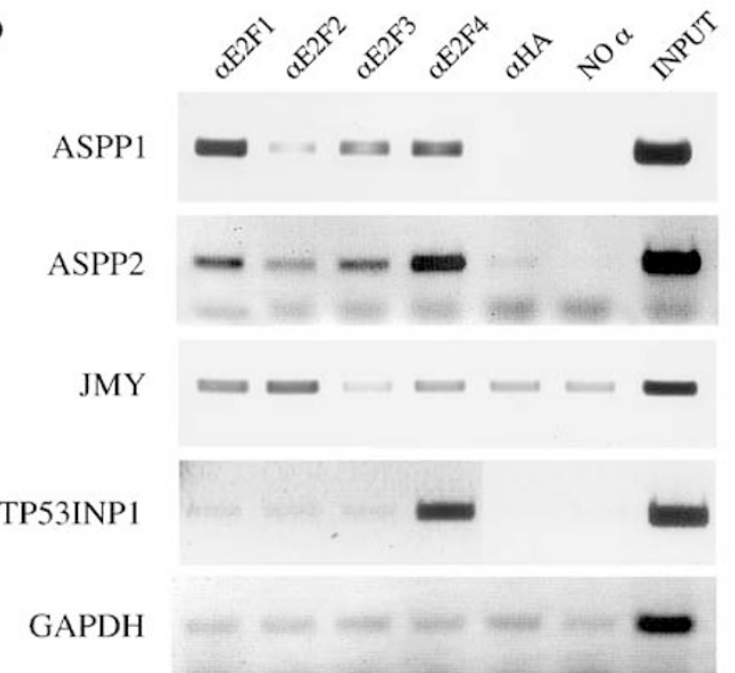

Figure 3 Endogenous E2Fs bind the promoters of ASPP1, ASPP2, JMY and TP53INP1. (a) Schematic representation of the upstream regions of the human ASPP1, ASPP2 and TP53INP1 genes. E2F-binding sites are presented as striped boxes and 8-mer nucleotide sequences. Transcription start sites are marked by an arrow. (b) ChIP analysis was performed using proliferating WI38 cells. Crosslinked chromatin was immunoprecipitated with antibodies to E2F1, E2F2, E2F3, E2F4, HA or without antibody (no $\alpha$ ). Immunoprecipitates from each sample were analyzed by PCR using primers specific for the ASPP1, ASPP2, JMY and TP53INP1 promoters and for a GAPDH genomic fragment. As a control, a sample representing $0.2 \%$ of the total chromatin used for IP reactions was included (input)

against E2F1, E2F2, E2F3 and E2F4. The promoter fragments that were amplified were: ASPP1, $-380 /+4$; ASPP2, -207/ + 16; JMY, -243/ + 1; TP53INP1, -415/-121. Endogenous E2F1 was found associated with all these promoters (Figure $3 \mathrm{~b}$ ). In addition, we detected binding of endogenous E2F3 and E2F4 to the ASPP1 and ASPP2 promoters, binding of E2F2 to the JMY promoter and strong binding of E2F4 to the TP53INP1 promoter (Figure 3b). No binding of endogenous E2Fs was detected to an unrelated genomic fragment (Figure $3 b$ ).

TP53INP1 interacts with a kinase that phosphorylates serine 46 of $p 53^{22,27}$; moreover, TP53INP1 induces phosphorylation of $\mathrm{p} 53$ on this residue. ${ }^{22}$ In agreement with the ability of E2F1 to induce TP53INP1 expression, we detected an increase in phosphorylation of p53 on serine 46 upon activation of E2F1 (Figure 4a). Such an increase was not detected with the DNA binding-deficient mutant of E2F1 (E2F1 E132; Figure 4a), indicating that the transcriptional activity of E2F1 is required for this phosphorylation, presumably through enhancement of TP53INP1 gene expression. 
a

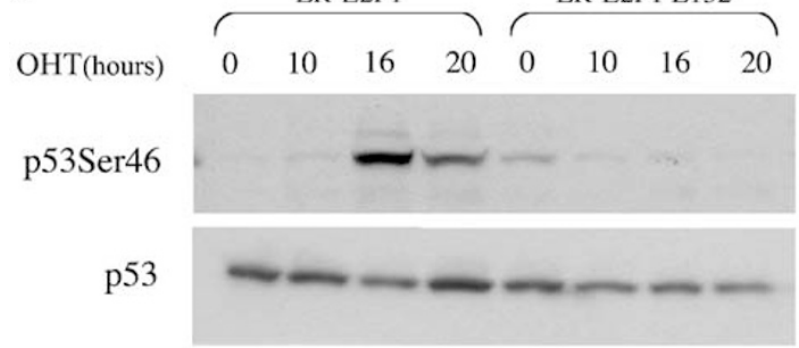

C

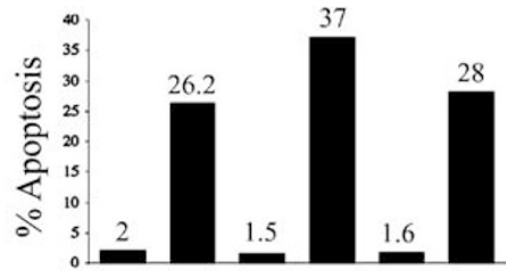

p53

OHT

wt p53

p53S46A b
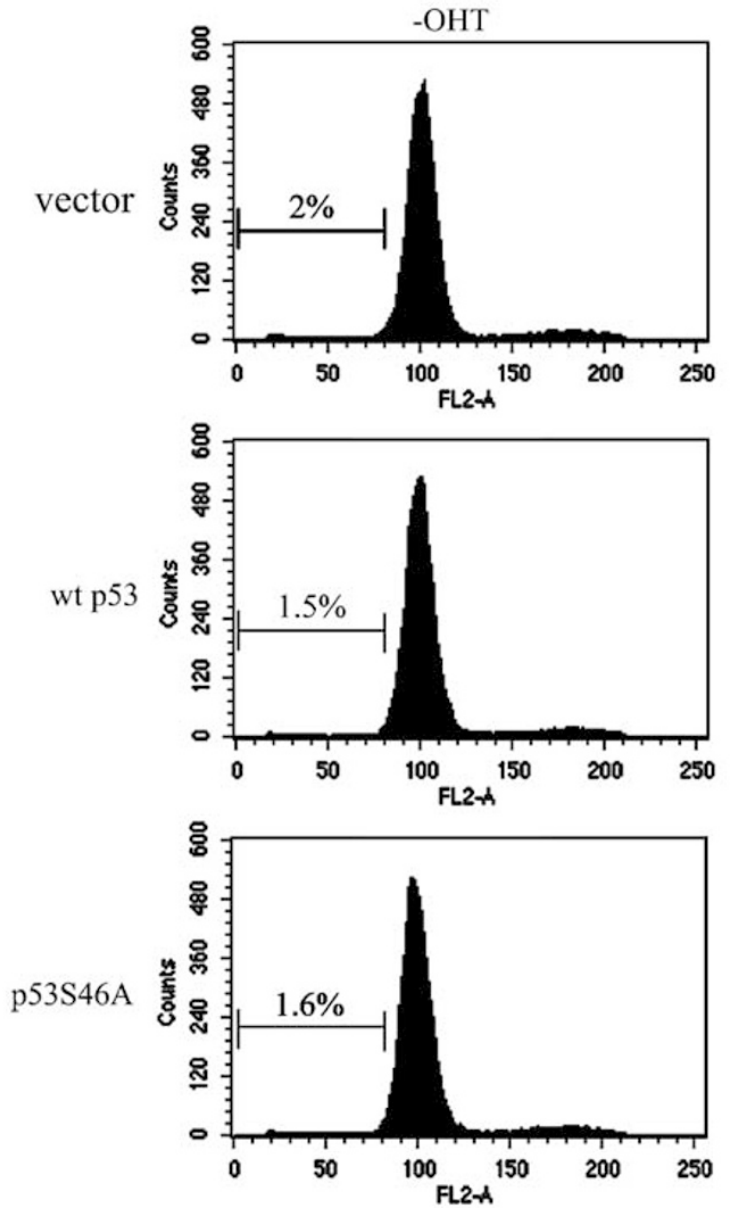
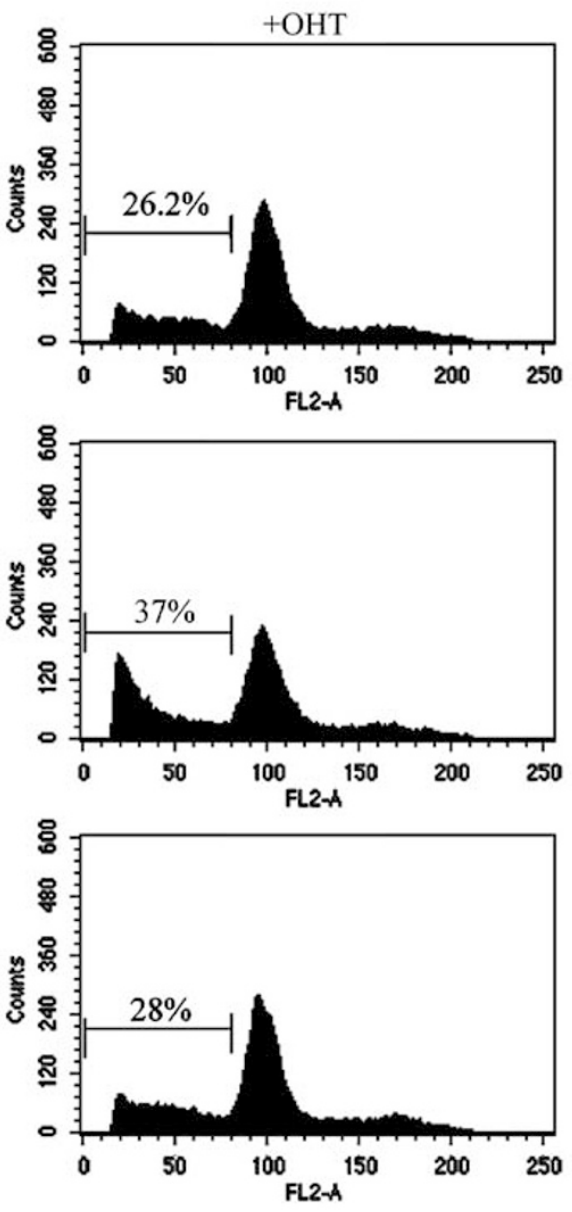

Figure 4 E2F1-induced phosphorylation of p53 on serine 46 is important for cooperation between E2F1 and p53 in apoptosis. (a) U2OS cells were infected with a retrovirus expressing either ER-wild type E2F1 (ER-E2F1) or ER-E2F1-E132. Infected cells were incubated with OHT (300 nM) for the times indicated at the top of each lane. Proteins were extracted from the cells and equal amounts of protein (determined by Bradford assay) were used for Western blot analysis with an antiPhospho-p53Ser46 antibody (upper panel) and anti-p53 antibody (lower panel, CM1). (b) Saos-2 cells stably expressing ER-E2F1 were infected with a control retrovirus (vector) or a retrovirus expressing either wt-p53 (wt p53) or the p53S46A p53 mutant. Infected cells were incubated with OHT ( $300 \mathrm{nM}$ ) for $76 \mathrm{~h}(+\mathrm{OHT}$ ) or left untreated $(-\mathrm{OHT})$, and then harvested for either FACS analysis (b) or protein extraction (c, lower panel). (c) The bar graph depicts the apoptotic rate of the samples shown in (b). The lower panel depicts Western blot analysis using an anti-p53 antibody (CM1)

E2F1 and p53 cooperate in apoptosis. ${ }^{3,4}$ However, in view of the growing number of functional links between E2F1 and p53, the relative contribution of each of these links to the E2F1-p53 cooperation is not entirely clear. We, therefore, tested the relevance of E2F1-induced phosphorylation of p53 on serine 46 to the E2F1-p53 cooperation in apoptosis. To this end, p53 and E2F1 were expressed either alone or together in p53-deficient human SAOS-2 cells and the ensuing apoptosis was measured. Expression of either wt p53 alone or mutant p53S46A, in which the serine at position 46 was substituted by alanine, did not cause apoptosis. Activation of ER-E2F1 resulted in significant apoptosis, as determined by the appearance of cells with sub-G1 DNA content (Figure 4b). Importantly, this E2F1-induced apoptosis 
was further enhanced by co-expression of wt p53, but not p53S46A (Figure $4 b$ and $c$ ). These data strongly suggest that phosphorylation of p53 on serine 46 plays an important role in E2F1-p53 cooperation in apoptosis. Of note, wt p53 and p53S46A were expressed at comparable levels and were similarly stabilized upon E2F1 activation (Figure 4c), indicating that stabilization of p53 by E2F1 is not sufficient for E2F1p53 cooperation in apoptosis.

\section{Discussion}

E2F and p53 were shown to cooperate in apoptosis 10 years ago; ${ }^{3,4}$ however, the mechanisms that mediate this cooperation are still not fully understood. One mechanism involves E2F-induced activation of genes whose protein products positively regulate $\mathrm{p} 53$ stability and transcriptional activity; this group is constantly growing and presently includes ARF, ATM, Chk2 and PIN1. ${ }^{15-17,30-36}$ After its stabilization and activation, p53 can elicit a number of different cellular responses including, most notably, growth arrest and apoptosis. The E2F-regulated genes listed above, while contributing to p53 activation, do not explain how E2F1 directs p53 to favor apoptosis over growth arrest. Such explanation is now provided by our finding that ectopic expression of E2F1 or deregulation of the $\mathrm{RB} / \mathrm{E} 2 \mathrm{~F}$ pathway result in coordinated upregulation of four proapoptotic cofactors of p53, ASPP1, ASPP2, JMY and TP53INP1 via a direct transcriptional mechanism.

ASPP1 and ASPP2 also induce apoptosis independently of p53 by binding to the p53 family members, p63 and p73. ${ }^{37}$ Therefore, their activation by E2F1 may play a role also in E2F1-induced p53-independent apoptosis.

As shown here, E2F1 upregulates the expression of TP53INP1. TP53INP1 was previously shown to induce phosphorylation of human p53 on serine $46^{22}$ and, indeed, we detect an E2F1-induced phosphorylation of p53 on serine 46. This covalent modification of p53 was reported to occur upon severe DNA damage and play an important role in p53mediated apoptosis. ${ }^{38}$ Of note, we and others demonstrated that E2F1 also induces phosphorylation of p53 on other residues that are similarly phosphorylated after damage, including serine 15 and serine $20 .^{11,15,16}$ E2F transcriptionally activates the kinases ATM and Chk2 and this most probably contributes to E2F-induced p53 phosphorylation on these residues. ${ }^{15,17}$ It remains to be determined whether E2F also regulates the expression of kinases such as HIPK2, which mediates p53 phosphorylation on serine $46 .{ }^{39,40}$

As shown here, E2F1-induced phosphorylation of p53 on serine 46 is required for E2F1-p53 cooperation in apoptosis, as evidenced by the fact that a mutant p53 incapable of undergoing phosphorylation at serine 46 fails to exhibit such cooperation. Interestingly, E2F1 still stabilizes this p53 mutant, indicating that E2F1-induced stabilization of $p 53$ is not sufficient for E2F1-p53 cooperation in apoptosis.

Upon its phosphorylation on serine 46, p53 was shown to transcriptionally activate the proapoptotic gene p53AIP1. ${ }^{38}$ In agreement with this report, we observe an increase in p53AIP1 mRNA levels after E2F1 activation. However, E2F1-induced elevation of p53AIP1 expression can also occur in p53-deficient cells (data not shown), suggesting that p53AIP1 may be independently regulated by both p53 and E2F1. Thus, E2F1 may regulate the expression of p53AIP1 both directly and indirectly, via p53. A number of additional genes, including Apaf-1, PUMA, Noxa and SIVA, were shown to be directly regulated by both $\mathrm{p} 53$ and $\mathrm{E} 2 \mathrm{~F} 1 \mathrm{H}^{41-48}$ Presumably, the cooperation between E2F1 and p53 in the activation of some proapoptotic genes leads to enhanced expression of those genes, also contributing to the E2F1-p53 cooperation in apoptosis.

In summary, E2F1-p53 cooperation in apoptosis may involve a number of parallel and perhaps synergistic mechanisms. First, E2F1 contributes to stabilization and general activation of p53 by transcriptionally activating ARF, ATM, Chk2 and PIN1; this, by itself, does not favor apoptosis or growth arrest. Second, E2F1 induces expression of the proapoptotic cofactors of p53, ASPP1, ASPP2, JMY and TP53INP1, thereby directing p53 to its proapoptotic targets. Thirdly, E2F1 directly activates some of the p53 proapoptotic target genes such as Apaf1, PUMA, Noxa, SIVA and probably also p53AIP1, thereby augmenting the upregulation of the corresponding transcripts by $\mathrm{p53}$. Additional studies are required to fully elucidate the combinatorial effects of these different mechanisms and the intricate network by which E2F1 affects the levels and activity of p53 and its family members.

\section{Materials and Methods}

\section{Plasmids and antibodies}

The following plasmids were described previously: pBabe-puro-HA-ERE2F1 and pBabe-puro-HA-ER-E2F1 E132, ${ }^{43}$ pBabe-puro-E2F1 ${ }^{15}$ pBabe-puro-E1A12S was a kind gift of $K$ Helin. pBabe-puro-p53 WT and pBabe-puro-p53 S46A were constructed by subcloning the inserts from $\mathrm{pC} 53-\mathrm{SN} 3$ and $\mathrm{pC} 53-\mathrm{SNS}-\mathrm{S} 46 \mathrm{~A},{ }^{38}$ respectively, into $\mathrm{pBabe-puro}$ vector following standard molecular cloning procedures.

The following antibodies were used for immunoblotting: anti-JMY (L-16) (Santa Cruz Biotechnology, sc-10027), anti-ASPP1, ${ }^{20}$ anti-p53 (CM1; Novocastra) and anti-p53 (Ser46) (Cell Signaling Technology, \#2521).

\section{Cell culture}

U2OS and Saos-2 osteosarcoma cells expressing the ecotropic receptor (EcotR) were cultured in DMEM plus 5\% FCS. EcotR-expressing H1299 cells were cultured in RPMI 1640 plus 5\% FCS. Early passage (passage 16-20) WI38 human embryonic lung fibroblasts and EcotR-expressing WI38 fibroblasts were grown in MEM plus 15\% FCS, 2 mM L-glutamine, $1 \mathrm{mM}$ sodium pyruvate and nonessential amino acids. Cells were maintained at $37^{\circ} \mathrm{C}$ in a humidified $8 \% \mathrm{CO}_{2}$-containing atmosphere. Retroviral infections were performed essentially as described. ${ }^{47}$

To activate ER-E2F1, cells were treated with 4-hydroxytamoxifen $(\mathrm{OHT})(300 \mathrm{nM})$ for the indicated duration. $\mathrm{CHX}$ was used at $10 \mu \mathrm{g} / \mathrm{ml}$.

\section{RT-PCR and Western blotting}

Total RNA was extracted from cells using the Tri Reagent method (MRC, TR-118). Semiquantitative RT-PCR was performed essentially as described. ${ }^{49}$ Primers specific for GAPDH, 5'-ACCACAGTCCATGCCAT CAC and 5'-TCCACCACCCTGTTGCTGTA, ASPP1, 5'-GAAATGA ACGTCCCATACCC and 5'-TTTCAGCAGACAGATTGCC, ASPP2, 
5'-AAATCTTCCCCAGCAAGCC and 5'-GCACTTTCTTCTCTTTCTCCC, JMY, 5'-ATAAATCCACTCCCATCCCC and 5'-CCCCTTTCCTTATTTGT GCC, TP53INP1, 5'-AAACCTTCTCATTGAACATCCC and 5'-CCATTGT GCTTGACTTGCC, were used in PCR amplifications. Western blot analysis was performed essentially as described. ${ }^{15}$

\section{Chromatin immunoprecipitation}

Chromatin immunoprecipitation was performed essentially as described. ${ }^{47}$ Antibodies to HA (Santa Cruz, sc-805), E2F1 (Santa Cruz, sc-193), E2F2 (Santa Cruz. Sc-633X), E2F3 (Santa Cruz, sc-879) and E2F4 (Santa Cruz, sc-1082 + sc-866) $(1.2 \mu \mathrm{g}$ per immunoprecipitation) were used to precipitate chromosomal DNA, employing crosslinked chromatin prepared from exponentially growing WI38 cells. The immunoprecipitated DNA was analyzed by PCR using the following primers: ASPP1, 5'-GCGGTCTTCC CTAGTCGG and 5'-CTCTCGCTGGCCCTGTCGC, ASPP2, 5'-AA GAAAAGGCGGCCGGCTCC and 5'-CTTCCGACGCTCGTGACGGTC, JMY, 5'-CTTGTGCTTATCCAAGAGCTG and 5' AATGGGAAGTGT TAAGGCTA, TP53INP1, 5'-AAACCCTCGACCCTTCACTC and 5'-CGA GAGGTTGTCACCAACG, GAPDH, 5'-GTATTCCCCCAGGTTTACAT and $5^{\prime}$-TTCTGTCTTCCACTCACTCC.

\section{FACS analysis}

Cells were trypsinized and fixed in $70 \%$ ethanol $\left(4^{\circ} \mathrm{C}\right.$, overnight). After fixation, cells were centrifuged for $5 \mathrm{~min}$ at $1200 \mathrm{rpm}$ and incubated for $30 \mathrm{~min}$ at $4^{\circ} \mathrm{C}$ in $1 \mathrm{ml}$ of PBS, centrifuged and resuspended in PBS containing $25 \mu \mathrm{g} / \mathrm{ml}$ propidium iodide and $50 \mu \mathrm{g} / \mathrm{ml}$ RNAse A for $20 \mathrm{~min}$ at room temperature. Fluorescence intensity was analyzed on a FACSCAN machine (Becton Dickinson).

\section{Acknowledgements}

We are grateful to Yocheved Lamed for excellent technical assistance. We thank Karen Vousden and Xin Lu for communicating results prior to publication. We also thank Xin Lu for antibodies to ASPP1 and ASPP2 and Yoichi Taya for the pC53-SNS-S46A plasmid. This work was supported by an Israel Science Foundation (ISF) grant to DG, and by grant R37 CA40099 from the National Cancer Institute to MO. MC was supported by a Pasteur-Weizmann post-doctoral fellowship.

\section{References}

1. DeGregori J (2002) The genetics of the E2F family of transcription factors: shared functions and unique roles. Biochim. Biophys. Acta 1602: 131-150

2. Ginsberg D (2002) E2F1 pathways to apoptosis. FEBS Lett. 529: 122-125

3. Qin XQ, Livingston DM, Kaelin Jr WG and Adams PD (1994) Deregulated transcription factor E2F-1 expression leads to S-phase entry and p53-mediated apoptosis. Proc. Natl. Acad. Sci. USA 91: 10918-10922

4. Wu X and Levine AJ (1994) p53 and E2F-1 cooperate to mediate apoptosis. Proc. Natl. Acad. Sci. USA 91: 3602-3606

5. Kowalik TF, DeGregori J, Schwarz JK and Nevins JR (1995) E2F1 overexpression in quiescent fibroblasts leads to induction of cellular DNA synthesis and apoptosis. J. Virol. 69: 2491-2500

6. Guy CT, Zhou W, Kaufman S and Robinson MO (1996) E2F-1 blocks terminal differentiation and causes proliferation in transgenic megakaryocytes. Mol. Cell. Biol. 16: 685-693

7. Holmberg C, Helin K, Sehested M and Karlstrom O (1998) E2F-1-induced p53independent apoptosis in transgenic mice. Oncogene 17: 143-155
8. Pierce AM, Fisher SM, Conti CJ and Johnson DG (1998) Deregulated expression of E2F1 induces hyperplasia and cooperates with ras in skin tumor development. Oncogene 16: 1267-1276

9. Field SJ, Tsai FY, Kuo F, Zubiaga AM, Kaelin WJ, Livingston DM, Orkin SH and Greenberg ME (1996) E2F-1 functions in mice to promote apoptosis and suppress proliferation. Cell 85: 549-561

10. Sherr CJ and Weber JD (2000) The ARF/p53 pathway. Curr. Opin. Genet. Dev. 10: $94-99$

11. Rogoff HA, Pickering MT, Debatis ME, Jones S and Kowalik TF (2002) E2F1 induces phosphorylation of p53 that is coincident with p53 accumulation and apoptosis. Mol. Cell. Biol. 22: 5308-5318

12. Russell JL, Powers JT, Rounbehler RJ, Rogers PM, Conti CJ and Johnson DG (2002) ARF differentially modulates apoptosis induced by E2F1 and Myc. Mol. Cell. Biol. 22: 1360-1368

13. Tolbert D, Lu X, Yin C, Tantama M and Van Dyke T (2002) p19(ARF) is dispensable for oncogenic stress-induced p53-mediated apoptosis and tumor suppression in vivo. Mol. Cell. Biol. 22: 370-377

14. Tsai KY, MacPherson D, Rubinson DA, Crowley D and Jacks T (2002) ARF is not required for apoptosis in $\mathrm{Rb}$ mutant mouse embryos. Curr. Biol. 12 159-163

15. Berkovich $E$ and Ginsberg D (2003) ATM is a target for positive regulation by E2F-1. Oncogene 22: 161-167

16. Powers JT, Hong S, Mayhew CN, Rogers PM, Knudsen ES and Johnson DG (2004) E2F1 uses the ATM signaling pathway to induce p53 and Chk2 phosphorylation and apoptosis. Mol. Cancer Res. 2: 203-214

17. Rogoff HA, Pickering MT, Frame FM, Debatis ME, Sanchez $Y$, Jones S and Kowalik TF (2004) Apoptosis associated with deregulated E2F activity is dependent on E2F1 and Atm/Nbs1/Chk2. Mol. Cell. Biol. 24: 29682977

18. Hiebert SW, Packham G, Strom DK, Haffner R, Oren M, Zambetti G and Cleveland JL (1995) E2F-1 : DP-1 induces p53 and overrides survival factors to trigger apoptosis. Mol. Cell. Biol. 15: 6864-6874

19. Ko LJ and Prives C (1996) p53: puzzle and paradigm. Genes Dev. 10: 1054-1072

20. Samuels-Lev Y, O'Connor DJ, Bergamaschi D, Trigiante G, Hsieh JK, Zhong S, Campargue I, Naumovski L, Crook T and Lu X (2001) ASPP proteins specifically stimulate the apoptotic function of p53. Mol. Cell 8: 781-794

21. Shikama N, Lee CW, France S, Delavaine L, Lyon J, Krstic-Demonacos M and La Thangue NB (1999) A novel cofactor for p300 that regulates the p53 response. Mol. Cell 4: 365-376

22. Okamura S, Arakawa H, Tanaka T, Nakanishi H, Ng CC, Taya Y, Monden M and Nakamura $Y$ (2001) p53DINP1, a p53-inducible gene, regulates p53dependent apoptosis. Mol. Cell 8: 85-94

23. Bergamaschi D, Samuels Y, O'Neil NJ, Trigiante G, Crook T, Hsieh JK, O'Connor DJ, Zhong S, Campargue I, Tomlinson ML, Kuwabara PE and Lu X (2003) iASPP oncoprotein is a key inhibitor of p53 conserved from worm to human. Nat. Genet. 33: 162-167

24. Slee EA, Gillotin S, Bergamaschi D, Royer C, Llanos S, Ali S, Jin B, Trigiante G and Lu X (2004) The N-terminus of a novel isoform of human iASPP is required for its cytoplasmic localization. Oncogene 23: 9007-9016

25. Tomasini R, Samir AA, Vaccaro MI, Pebusque MJ, Dagorn JC, lovanna JL and Dusetti NJ (2001) Molecular and functional characterization of the stressinduced protein (SIP) gene and its two transcripts generated by alternative splicing. SIP induced by stress and promotes cell death. J. Biol. Chem. 276: 44185-44192

26. Tomasini R, Samir AA, Pebusque MJ, Calvo EL, Totaro S, Dagorn JC, Dusetti $\mathrm{NJ}$ and lovanna JL (2002) P53-dependent expression of the stress-induced protein (SIP). Eur. J. Cell Biol. 81: 294-301

27. Tomasini R, Samir AA, Carrier A, Isnardon D, Cecchinelli B, Soddu S, Malissen B, Dagorn JC, lovanna JL and Dusetti NJ (2003) TP53INP1s and homeodomain-interacting protein kinase-2 (HIPK2) are partners in regulating p53 activity. J. Biol. Chem. 278: 37722-37729

28. Vigo $E$, Muller $H$, Prosperini $E$, Hateboer $G$, Cartwright $P$, Moroni $M C$ and Helin $\mathrm{K}$ (1999) CDC25A phosphatase is a target of E2F and is required for efficient E2F-induced S phase. Mol. Cell. Biol. 19: 6379-6395

29. Weinmann AS, Yan PS, Oberley MJ, Huang TH and Farnham PJ (2002) Isolating human transcription factor targets by coupling chromatin immunoprecipitation and CpG island microarray analysis. Genes Dev. 16: 235-244 
30. Bates S, Phillips AC, Clark PA, Stott F, Peters G, Ludwig RL and Vousden KH (1998) p14ARF links the tumour suppressors RB and p53 (letter). Nature 395 124-125

31. Chehab NH, Malikzay A, Appel M and Halazonetis TD (2000) Chk2/hCds functions as a DNA damage checkpoint in $\mathrm{G}(1)$ by stabilizing p53. Genes Dev. 14: $278-288$

32. Hirao A, Kong YY, Matsuoka S, Wakeham A, Ruland J, Yoshida H, Liu D, Elledge SJ and Mak TW (2000) DNA damage-induced activation of p53 by the checkpoint kinase Chk2. Science 287: 1824-1827

33. Shieh SY, Ahn J, Tamai K, Taya Y and Prives C (2000) The human homologs of checkpoint kinases Chk1 and Cds1 (Chk2) phosphorylate p53 at multiple DNA damage-inducible sites. Genes Dev. 14: 289-300

34. Ryo A, Liou YC, Wulf G, Nakamura M, Lee SW and Lu KP (2002) PIN1 is an E2F target gene essential for Neu/Ras-induced transformation of mammary epithelial cells. Mol. Cell. Biol. 22: 5281-5295

35. Zacchi $P$, Gostissa M, Uchida T, Salvagno C, Avolio F, Volinia S, Ronai Z, Blandino G, Schneider C and Del Sal G (2002) The prolyl isomerase Pin1 reveals a mechanism to control p53 functions after genotoxic insults. Nature 419: 853-857

36. Zheng H, You H, Zhou XZ, Murray SA, Uchida T, Wulf G, Gu L, Tang X, Lu KP and Xiao ZX (2002) The prolyl isomerase Pin1 is a regulator of p53 in genotoxic response. Nature 419: 849-853

37. Bergamaschi D, Samuels Y, Jin B, Duraisingham S, Crook T and Lu X (2004) ASPP1 and ASPP2: common activators of p53 family members. Mol. Cell. Biol. 24: $1341-1350$

38. Oda K, Arakawa H, Tanaka T, Matsuda K, Tanikawa C, Mori T, Nishimori H Tamai K, Tokino T, Nakamura Y and Taya Y (2000) p53AIP1, a potential mediator of p53-dependent apoptosis, and its regulation by Ser-46phosphorylated p53. Cell 102: 849-862

39. Hofmann TG, Moller A, Sirma $H$, Zentgraf $H$, Taya $Y$, Droge $W$, Will $H$ and Schmitz ML (2002) Regulation of p53 activity by its interaction with homeodomain-interacting protein kinase-2. Nat. Cell Biol. 4: 1-10
40. D'Orazi G, Cecchinelli B, Bruno T, Manni I, Higashimoto Y, Saito S, Gostissa M, Coen S, Marchetti A, Del Sal G, Piaggio G, Fanciulli M, Appella E and Soddu S (2002) Homeodomain-interacting protein kinase-2 phosphorylates p53 at Ser 46 and mediates apoptosis. Nat. Cell Biol. 4: 11-19

41. Oda E, Ohki R, Murasawa H, Nemoto J, Shibue T, Yamashita T, Tokino T, Taniguchi T and Tanaka N (2000) Noxa, a BH3-only member of the Bcl-2 family and candidate mediator of p53-induced apoptosis. Science 288: 1053-1058

42. Guo Z, Yikang S, Yoshida H, Mak TW and Zacksenhaus E (2001) Inactivation of the retinoblastoma tumor suppressor induces apoptosis protease-activating factor-1 dependent and independent apoptotic pathways during embryogenesis. Cancer Res. 61: 8395-8400

43. Moroni MC, Hickman ES, Denchi EL, Caprara G, Colli E, Cecconi F, Muller H and Helin K (2001) Apaf-1 is a transcriptional target for E2F and p53. Nat. Cell Biol. 3: $552-558$

44. Nakano K and Vousden KH (2001) PUMA, a novel proapoptotic gene, is induced by $p 53$. Mol. Cell 7: 683-694

45. Robles Al, Bemmels NA, Foraker AB and Harris CC (2001) APAF-1 is a transcriptional target of $p 53$ in DNA damage-induced apoptosis. Cancer Res. 61: 6660-6664

46. Yu J, Zhang L, Hwang PM, Kinzler KW and Vogelstein B (2001) PUMA induces the rapid apoptosis of colorectal cancer cells. Mol. Cell 7: 673-682

47. Hershko $\mathrm{T}$ and Ginsberg D (2004) Up-regulation of Bcl-2 homology 3 (BH3)-only proteins by E2F1 mediates apoptosis. J. Biol. Chem. 279: 8627-8634

48. Fortin A, MacLaurin JG, Arbour N, Cregan SP, Kushwaha N, Callaghan SM, Park DS, Albert PR and Slack RS (2004) The proapoptotic gene SIVA is a direct transcriptional target for the tumor suppressors p53 and E2F1. J. Biol. Chem. 279: 28706-28714

49. Kalma $Y$, Marash L, Lamed $Y$ and Ginsberg D (2001) Expression analysis using DNA microarrays demonstrates that E2F-1 up-regulates expression of DNA replication genes including replication protein A2. Oncogene 20: 1379-1387 